\title{
Ahijamiento de Lolium perenne L. asociado con Dactylis glomerata L. y Trifolium repens L.
}

\section{Tillering of Lolium perenne L. associated with Dactylis glomerata L. and Trifolium repens L.}

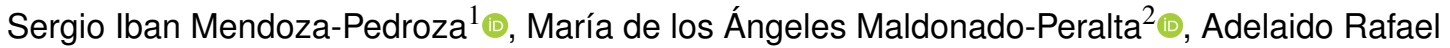 \\ Rojas-García ${ }^{2 *}$ (D), Aldenamar Cruz-Hernández ${ }^{3}$ (D), Nicolás Torres-Salado², Humberto Vaquera-Huerta ${ }^{2}$ (D) \\ ${ }^{1}$ Campus Montecillo. Colegio de Postgraduados. Carretera México-Texcoco Km. 36.5, Montecillo, Texcoco. CP. 56230. Estado de \\ México, México. \\ ${ }^{2}$ Facultad de Medicina Veterinaria y Zootecnia No 2. Universidad Autónoma de Guerrero. CP. 41940. Cuajinicuilapa, Guerrero, México. \\ ${ }^{3}$ División Académica de Ciencias Agropecuarias. Universidad Juárez Autónoma de Tabasco, km 25. Carretera Villahermosa-Teapa, R/A \\ La Huasteca, Mpio de Centro, Tabasco, México. \\ *Autor de correspondencia: rogarcia@uagro.com
}

Artículo científico recibido: 07 de marzo de 2018 aceptado: 21 de noviembre de 2018

RESUMEN. El objetivo del presente estudio fue evaluar el peso, tasa de aparición, muerte, sobrevivencia de tallos de ballico perenne en monocultivo y asociado con pasto ovillo y trébol blanco. El mayor peso por tallo en ballico perenne ocurrió en monocultivo con $0.49 \mathrm{~g}$ tallo $^{-1}$ y menor en la asociación $20: 40: 40$ con $0.36 \mathrm{~g}$ tallo ${ }^{-1}$. Para el pasto ovillo el mayor peso fue en asociación 70:20:10 con $0.16 \mathrm{~g}_{\text {tallo }}{ }^{-1}$. La mayor tasa de aparición y muerte de tallos del pasto ballico, se presentó en ballico en monocultivo (100:00:00) con 0.16 y 0.30 tallos $^{*} 100$ tallos día ${ }^{-1}$, respectivamente. La tasa de sobrevivencia de tallo ballico perenne fue mayor en otoño y menor en verano con 0.87 y 0.76 tallos $^{*} 100$ tallos día ${ }^{-1}$, respectivamente. La tasa de aparición y muerte de tallos aumento conforme transcurrió la investigación de 0.08 en otoño a 0.18 tallos 100 tallos día ${ }^{-1}$ en verano. De forma inversa, la sobrevivencia en ovillo disminuyo con el tiempo siendo en otoño mayor con 0.92 y verano menor con 0.78 tallos $^{*} 100$ tallos día ${ }^{-1}$. El pasto ballico perenne fue la especie que mayor mortalidad de tallos presento, siendo mayor la afectación cuando se encuentra en monocultivo.

Palabras clave: Asociaciones, estructurales, gramíneas, leguminosa, morfológicos.

ABSTRACT. The aim of this study was to evaluate the weight and appearance, death and survival rates of perennial ryegrass stems in monoculture and associated with orchard grass and white clover. The highest weight per stem in perennial ryegrass occurred in monoculture with $0.49 \mathrm{~g} \mathrm{stem}^{-1}$ and lowest in the 20:40:40 association with $0.36 \mathrm{~g} \mathrm{stem}^{-1}$. For orchard grass, the greatest weight was in the 70:20:10 association with $0.16 \mathrm{~g} \mathrm{stem}^{-1}$. The highest ryegrass stem appearance and death rates occurred in ryegrass in monoculture (100:00:00) with 0.16 and 0.30 stems $^{*} 100$ stems day $^{-1}$, respectively. The perennial ryegrass stem survival rate was higher in autumn and lower in summer with 0.87 and 0.76 stems $^{*} 100$ stems day $^{-1}$, respectively. The stem appearance and death rates increased as the research progressed from 0.08 in autumn to 0.18 stems ${ }^{*} 100$ stems day $^{-1}$ in winter. Conversely, the survival in orchard grass decreased with time, being higher in autumn with 0.92 and lower in summer with 0.78 stems $^{*} 100$ stems day $^{-1}$. Perennial ryegrass was the species with the highest stem mortality, being more affected when in monoculture.

Key words: Associations, structural, grasses, legume, morphological.

\section{INTRODUCCIÓN}

Las especies forrajeras que más se cultivan en las regiones templadas de México es el ballico perenne (Lolium perenne L.) y pasto ovillo (Dactylis glomerata L.), que se utilizan en pastoreo con anima- les diversos, por su adaptación al clima y facilidad para crecer en diferentes tipos de suelos (Hernández et al. 2015, Maldonado et al. 2017). Pero para tener alto rendimiento y calidad en una especie es indispensable el aporte de fertilizante nitrogenados, lo que aumenta los costos de producción (Grünwaldt et al. 
2015). La utilización de leguminosas en la pradera, es una alternativa para la producción sostenible (Lüscher et al. 2014). Asociando gramíneas con leguminosas como el trébol blanco (Trifolium repens L.), la estacionalidad disminuye y aumenta el rendimiento y la calidad, ya que las leguminosas forman nódulos en los cuales se alojan las bacterias del género Rhizobium y fijan el nitrógeno atmosférico al suelo, lo que es aprovechado por las raíces de las gramíneas (Rojas et al. 2016a, 2016b).

La dinámica de ahijamiento y peso de tallos se ha evaluado en varias partes del mundo, por ser indicadores de la producción de forraje (Matthew et al. 1996). La producción de forraje en una pradera puede dividirse en dos componentes: la densidad de tallos por unidad de área y el rendimiento individual por tallo (Hernández et al. 2000, Rojas et al. 2017a). Por lo que la persistencia y producción de las especies forrajeras depende del balance entre tamaño/densidad (Hernández et al. 1999). En el desarrollo de una pradera, los tallos continuamente emergen, crecen y mueren en tasas que difieren dependiendo del manejo, condiciones ambientales y estado de desarrollo (Berone 2016).

El crecimiento vegetativo de una pradera depende de las características morfogenéticas: aparición de hojas, elongación y vida media (Arredondo y Schnyder 2003). La combinación de estas tres características determina el índice de área foliar de la pradera, factor principal para la intercepción de luz y la dinámica de tallos (Velasco et al. 2002). La tasa de crecimiento de una pradera es la integral de las tasas de crecimiento de los tallos individuales y por la tasa de aparición y muerte de los mismos (Carneiro et al. 2008).

En el valle de México se evaluaron diferentes asociaciones con pasto ovillo, ballico perenne y trébol blanco, y se encontró mayor peso por tallo en las estaciones de primavera y verano relacionado con la mayor temperatura y redimiento. Se encontró que las tasas de aparición, muerte y sobrevivencia de tallos están en constante cambio y depende de la proporción de cada especie en la mezcla, tiempo de establecida en la pradera y manejo (Rojas et al. 2016a, Maldonado et al. 2017, Rojas et al. 2017b).
Al respecto, Castro et al. (2013) reporta que estas mismas especies en asociación se tiene mayor peso por tallo en ballico perenne con $0.38 \mathrm{~g}^{\text {tallo }}{ }^{-1}$ en verano y la mayor densidad de tallos en invierno (9 961 tallos $\mathrm{m}^{-2}$ ). En México se tienen pocos ensayos de dinámica de tallos, que ayuden a entender la importancia de la tasa de aparición, muerte, sobrevivencia y componentes del rendimiento de cualquier especie forrajera. El conocimiento de la dinámica poblacional de tallos durante el año es una referencia esencial para el manejo de praderas. Con base a lo anterior, el objetivo del presente estudio fue evaluar el peso, tasa de aparición, muerte, sobrevivencia de tallos de ballico perenne y pasto ovillo asociados con trébol blanco y monocultivo de ballico perenne.

\section{MATERIALES Y MÉTODOS}

El ensayo se realizó durante un año, en el Campo Experimental del Colegio de Postgraduados, Montecillo, Texcoco, Estado de México, ubicado a $19^{\circ}$ $29^{\prime} \mathrm{N}$ y $98^{\circ} 53^{\prime} \mathrm{O}$, a una altura de 2240 metros sobre nivel del mar. El clima del lugar es templado subhúmedo, el más seco de los subhúmedos, con precipitación media anual de $636.5 \mathrm{~mm}$, régimen de lluvias en verano (junio a octubre), y temperatura media anual de $15.2{ }^{\circ} \mathrm{C}$ (García 2004). Se realizó un análisis del suelo en el Laboratorio de Nutrición Vegetal, obteniendo un suelo franco arenoso, con $\mathrm{N}, \mathrm{P}_{2} \mathrm{O}_{5}$, $\mathrm{K}_{2} \mathrm{O}$ de 3.4, 94 y $1337 \mathrm{ppm}$, respectivamente, $\mathrm{pH}$ de 8.4 , y $3.5 \%$ de materia orgánica.

Las praderas se establecieron el mes de febrero, mediante el método de hileras a $30 \mathrm{~cm}$ (gramíneas), mientras que la leguminosa fue en forma perpendicular con distancia entre surcos de 30 $\mathrm{cm}$; tomando como base las densidades de siembra 30,20 y $5 \mathrm{~kg} \mathrm{ha}^{-1}$ para ballico perenne, ovillo y trébol blanco, respectivamente. Las praderas no se fertilizaron y en las estaciones de otoño e invierno, se proporcionaron riegos a capacidad de campo cada dos semanas.

Antes de iniciar la investigación, se realizó un pastoreo de uniformización con ovinos, cosechando aproximadamente a $5 \mathrm{~cm}$ sobre el nivel del suelo. Posteriormente los pastoreos se realizaron cada 
cuatro semanas en primavera-verano y de 5 a 6 semanas en otoño-invierno, respectivamente. Los ovinos únicamente se utilizaron como defoliadores, los cuales fueron manejados en las parcelas experimentales con cerco eléctrico.

Los tratamientos fueron: 100:00:00, 70:20:10, 50:00:50, $40: 20: 40$ y 20:40:40\% de ballico perenne $(\mathrm{BP})$, pasto ovillo $(\mathrm{O})$ y trébol blanco $(\mathrm{TB})$, respectivamente. Se distribuyeron de forma aleatoria en 15 parcelas experimentales de 9 por $8 \mathrm{~m}$, bajo un diseño de bloques completos al azar con tres repeticiones (Hernández et al. 2015, Rojas et al. 2017a).

\section{Peso por tallo}

Un día antes de cada pastoreo, se cosecharon a ras de suelo 10 tallos con sus respectivas hojas de ballico perenne y pasto ovillo, los tallos se secaron en una estufa de aire forzado (Memmert Modelo UF 260) hasta peso constante, y posteriormente se registró el peso seco en una balanza de precisión (Sartorius Modelo Entris 323-S). El peso por tallo se obtuvo pesando los diez tallos y dividiendo entre el total.

\section{Tasa de aparición, muerte y sobrevivencia de tallos}

Para determinar la aparición y muerte de tallos, al inicio del experimento, en cada unidad experimental, se colocaron dos aros de plástico de $10.4 \mathrm{~cm}$ de diámetro, cuando las praderas fueron de la asociación de los dos pastos, cada aro delimitó un macollo de una especie y la pradera en monocultivo los aros delimitaban dos macollos de ballico perenne. Todos los tallos presentes dentro del aro se marcaron con anillos de cable de un mismo color, que se consideraron como población inicial. Posteriormente, cada mes, durante un año, los tallos nuevos se marcaron con anillos de diferente color, para cada generación y los tallos muertos se contaron y se les retiró el anillo.

Los valores de aparición y muerte de tallos se multiplicaron por el número de plantas $\mathrm{m}^{-2}$, con esos datos se estimó: la densidad poblacional de tallos (DPT; tallos $\mathrm{m}^{-2}$ ) y sus respectivas tasas mensuales de aparición (TAT) y muerte (TMT) (\%). Donde: DPT $=$ No. De tallos vivos existentes en cada muestreo.

$$
\begin{aligned}
\text { TAT } & =\frac{\text { No. de tallos nuevos }}{D T \text { del muestreo anterior }}(100) \\
\text { TMT } & =\frac{\text { No. de tallos muertos }}{D T \text { del muestreo anterior }}(100)
\end{aligned}
$$

La tasa de sobrevivencia de tallos (TST) se obtuvo de manera indirecta mediante la ecuación:

$$
\mathrm{TST}=1-\mathrm{TMT}
$$

\section{Datos climatológicos}

La temperatura máxima mensual oscilo entre 28 y $36^{\circ} \mathrm{C}$, en tanto que la temperatura mínima mensual fue de -13 y $6{ }^{\circ} \mathrm{C}$. Las mayores temperaturas se presentaron en primavera y verano, y fueron superior a $25{ }^{\circ} \mathrm{C}$, registrando la máxima temperatura en abril y mayo con $36{ }^{\circ} \mathrm{C}$. La menor temperatura se registró a finales de otoño e inicios de invierno con $-13{ }^{\circ} \mathrm{C}$ en noviembre y diciembre. La precipitación acumulada de agosto a septiembre fue de $518.5 \mathrm{~mm}$, de los cuales el $57 \%$ se registraron en el mes de agosto (Figura 1).

\section{Análisis estadístico}

Para comparar el efecto de las asociaciones estudiadas, se realizó un análisis de varianza con el procedimiento de Modelos Mixtos (SAS 2009). La comparación de medias se realizó con la prueba de Tukey ajustada $(\alpha=0.05)$.

\section{RESULTADOS}

\section{Peso por tallo}

En la Tabla 1 se presentan los cambios estacionales en el peso por tallo (g) del pasto ballico perenne en monocultivo y asociado. Se observaron diferencias estadísticas $(p=0.05)$ en los promedios anuales entre tratamientos. El mayor y menor valor se registró en el ballico perenne en monocultivo y en asociación 20:40:40 de BP:O:TB con 0.49 y $0.36 \mathrm{~g}$ tallo $^{-1}$, respectivamente. Independientemente de las asociaciones se observó una tendencia de reducir el peso por tallo, conforme transcurrió el experimento con el siguiente orden. otoño, invierno, primavera y verano con $0.64,0.55,0.43$ y $0.16 \mathrm{~g}$ tallo $^{-1}$, respectivamente $(p=0.05)$. 
Tabla 2. Tasa de aparición, muerte y sobrevivencia de tallos de ballico perenne (Lolium perenne L.) asociado con pasto ovillo (Dactylis glomerata L.) y trébol blanco (Trifolium repens L.).

\begin{tabular}{|c|c|c|c|c|c|c|c|}
\hline Asociación BP:O:TB & Otoño & Invierno & Primavera & Verano & EEM & Sig. & Promedio \\
\hline \multicolumn{8}{|c|}{ Tasa de aparición de tallos (Tallos * 100 tallos día $^{-1}$ ) } \\
\hline $100: 00: 00$ & $0.14^{b}$ & $0.15^{b}$ & $0.16^{b}$ & $0.19^{a}$ & 0.09 & ** & $0.16^{A}$ \\
\hline $70: 20: 10$ & $0.13^{b}$ & $0.14^{b}$ & $0.15^{a b}$ & $0.16^{a}$ & 0.08 & * & $0.15^{A B}$ \\
\hline $50: 00: 50$ & $0.12^{b}$ & $0.13^{b}$ & $0.14^{a b}$ & $0.15^{a}$ & 0.09 & * & $0.14^{B}$ \\
\hline 40:20:40 & $0.11^{b}$ & $0.12^{b}$ & $0.14^{a b}$ & $0.15^{a}$ & 0.07 & * & $0.13^{B}$ \\
\hline $20: 40: 40$ & $0.1^{b}$ & $0.12^{a b}$ & $0.13^{a b}$ & $0.14^{a}$ & 0.07 & * & $0.12^{B}$ \\
\hline Promedio & $0.12^{b}$ & $0.13^{b}$ & $0.14^{a b}$ & $0.16^{a}$ & 0.08 & * & \\
\hline \multicolumn{8}{|c|}{ Tasa de muerte de tallos (Tallos * 100 tallos día ${ }^{-1}$ ) } \\
\hline $100: 00: 00$ & $0.22^{c}$ & $0.27^{b}$ & $0.31^{a b}$ & $0.4^{a}$ & 0.08 & ** & $0.30^{A}$ \\
\hline $70: 20: 10$ & $0.14^{c}$ & $0.17^{b}$ & $0.22^{a b}$ & $0.27^{a}$ & 0.07 & ** & $0.20^{B}$ \\
\hline $50: 00: 50$ & $0.12^{c}$ & $0.13^{c}$ & $0.16^{b}$ & $0.19^{a}$ & 0.04 & * & $0.15^{C}$ \\
\hline $40: 20: 40$ & $0.12^{c}$ & $0.14^{c}$ & $0.16^{b}$ & $0.18^{a}$ & 0.05 & * & $0.15^{C}$ \\
\hline $20: 40: 40$ & $0.11^{c}$ & $0.13^{b}$ & $0.14^{b}$ & $0.17^{a}$ & 0.07 & ** & $0.14^{C}$ \\
\hline Promedio & $0.14^{c}$ & $0.17^{b}$ & $0.2^{b}$ & $0.24^{a}$ & 0.07 & ** & \\
\hline \multicolumn{8}{|c|}{ Tasa de sobrevivencia de tallos (TST) } \\
\hline $100: 00: 00$ & $0.78^{a}$ & $0.73^{a b}$ & $0.69^{a b}$ & $0.6^{b}$ & 0.08 & * & $0.70^{B}$ \\
\hline $70: 20: 10$ & $0.86^{a}$ & $0.84^{a b}$ & $0.78^{b}$ & $0.73^{c}$ & 0.09 & ** & $0.80^{A B}$ \\
\hline $50: 00: 50$ & $0.88^{a}$ & $0.87^{a}$ & $0.84^{a b}$ & $0.81^{b}$ & 0.07 & ** & $0.85^{A}$ \\
\hline $40: 20: 40$ & $0.88^{a}$ & $0.86^{a}$ & $0.84^{a b}$ & $0.82^{b}$ & 0.08 & ** & $0.85^{A}$ \\
\hline $20: 40: 40$ & $0.89^{a}$ & $0.87^{a}$ & $0.86^{a}$ & $0.83^{b}$ & 0.07 & * & $0.86^{A}$ \\
\hline Promedio & $0.87^{a}$ & $0.83^{a b}$ & $0.81^{a b}$ & $0.76^{b}$ & 0.08 & * & \\
\hline
\end{tabular}

0.05) entre asociaciones y estaciones del año (Tabla 2). La mayor tasa de mortalidad se presentó en la especie pura de ballico perenne con 0.30 tallos* 100 tallos día ${ }^{-1}$, mientras que las menores en las asociaciones 50:00:50, 40:20:40 y 20:40:40 de BP:O:TB, con promedio de 0.15 tallos $^{*} 100$ tallos día ${ }^{-1}$. Independientemente de los tratamientos, se observó un incremento en la mortalidad de tallos conforme avanzó el estudio, mostrándose los valores más altos en verano con 0.24 tallos $^{*} 100$ tallos día $^{-1}$, y los más bajos en otoño con 0.12 tallos $^{*} 100$ tallos día $^{-1}$. La mayor tasa de sobrevivencia se presentó en las asociaciones y menor en ballico perenne en monocultivo con 0.84 y 0.70 tallos $^{*} 100$ tallos día $^{-1}$, respectivamente. Independientemente de los tratamientos, los promedios estacionales muestran una tendencia a reducir la sobrevivencia de tallos. El promedio estacional para los diferentes tratamientos fue de $0.87 \mathrm{y}$ $0.76(p=0.05)$ para otoño y verano, respectivamente.

Por otra parte, la dinámica de ahijamiento, aparición de tallos, de pasto ovillo asociado con ballico perenne y trébol blanco (Tabla 3), indica dife- rencias estadísticas $(p=0.05)$ en los promedios anuales entre asociaciones. En todas las asociaciones se observó una tendencia a incrementarse la tasa de aparición de tallos con el transcurso del experimento. El menor y mayor valor se registró en otoño y verano con 0.08 y 0.17 tallos * 100 tallos día $^{-1}$. De manera similar, en la tasa de muerte en pasto ovillo (Tabla 3), no se presentaron diferencias estadísticas $(p=0.05)$ en los promedios anuales entre asociaciones con 0.13 tallos * 100 tallos día $^{-1}$. En todas las asociaciones la mortalidad se incrementó a través del estudio, siendo menor en otoño y mayor en verano $(p=0.05)$, con valores de 0.09 y 0.19 tallos * 100 tallos día $^{-1}$, respectivamente.

La tasa de sobrevivencia del pasto ovillo no presento diferencias estadísticas $(p=0.05)$ en los promedios anuales entre asociaciones con un promedio de 0.87 tallos $^{*} 100$ tallos día ${ }^{-1}$. Independientemente de las asociaciones, la sobrevivencia de tallos de pasto ovillo, presentó una tendencia a disminuir, siendo mayor $(p=0.05)$ en otoño con 0.91 y menor en verano con 0.81 tallos $^{*} 100$ tallos día $^{-1}$. 
Tabla 3. Tasa de aparición, muerte y sobrevivencia de tallos de pasto ovillo (Dactylis glomerata L.) asociado con ballico perenne (Lolium perenne L.) y trébol blanco (Trifolium repens L.).

\begin{tabular}{|c|c|c|c|c|c|c|c|}
\hline Asociación BP:O:TB & Otoño & Invierno & Primavera & Verano & EEM & Sig. & Promedio \\
\hline \multicolumn{8}{|c|}{ Tasa de aparición de tallos (Tallos * 100 tallos día ${ }^{-1}$ ) } \\
\hline $70: 20: 10$ & $0.08^{b}$ & $0.11^{b}$ & $0.12^{a b}$ & $0.15^{a}$ & 0.09 & * & 0.12 \\
\hline $40: 20: 40$ & $0.09^{b}$ & $0.11^{b}$ & $0.12^{b}$ & $0.16^{a}$ & 0.08 & ** & 0.12 \\
\hline $20: 40: 40$ & $0.08^{c}$ & $0.11^{b c}$ & $0.13^{b}$ & $0.18^{a}$ & 0.07 & ** & 0.13 \\
\hline Promedio & $0.08^{c}$ & $0.11^{b c}$ & $0.12^{b}$ & $0.17^{a}$ & 0.08 & ** & \\
\hline \multicolumn{8}{|c|}{ Tasa de muerte de tallos (Tallos * 100 tallos día ${ }^{-1}$ ) } \\
\hline $70: 20: 10$ & $0.08^{c}$ & $0.10^{c}$ & $0.14^{b}$ & $0.22^{a}$ & 0.08 & ** & 0.14 \\
\hline $40: 20: 40$ & $0.10^{c}$ & $0.11^{c}$ & $0.13^{b}$ & $0.18^{a}$ & 0.09 & ** & 0.13 \\
\hline $20: 40: 40$ & $0.08^{c}$ & $0.10^{c}$ & $0.14^{b}$ & $0.18^{a}$ & 0.07 & ** & 0.13 \\
\hline Promedio & $0.09^{c}$ & $0.11^{c}$ & $0.13^{b}$ & $0.19^{a}$ & 0.08 & ** & \\
\hline \multicolumn{8}{|c|}{ Tasa de sobrevivencia de tallos (TST) } \\
\hline $70: 20: 10$ & $0.92^{a}$ & $0.9^{a}$ & $0.86^{a b}$ & $0.79^{b}$ & 0.08 & * & 0.87 \\
\hline $40: 20: 40$ & $0.9^{a}$ & $0.89^{a}$ & $0.87^{a}$ & $0.82^{b}$ & 0.09 & * & 0.87 \\
\hline $20: 40: 40$ & $0.92^{a}$ & $0.9^{a}$ & $0.85^{a b}$ & $0.81^{b}$ & 0.04 & ** & 0.87 \\
\hline Promedio & $0.91^{a}$ & $0.89^{a}$ & $0.86^{a b}$ & $0.81^{b}$ & 0.05 & $\star \star$ & \\
\hline
\end{tabular}

$\mathrm{abc}=$ Medias con la misma literal minúscula en una misma hilera. no son diferentes $(p=0.05)$; $A B C=$ Medias con la misma literal mayúscula en una misma columna. no son diferentes $(p=0.05)$; $\mathrm{BP}=$ ballico perenne; $\mathrm{O}=$ pasto ovillo; $\mathrm{TB}=$ trébol blanco; $\mathrm{EEM}=$ error estándar de la media; Sig $=$ Significativo $\left({ }^{\star} p=0.05,{ }^{* *} p=0.01\right)$.

\section{DISCUSIÓN}

\section{Peso por tallo}

El menor peso por tallo de ballico perenne se presentó en verano y el mayor en otoño, posiblemente influyó la mayor y menor temperatura registrada en estas estaciónes (Figura 1), al ser el ballico una especie de clima templado frio (Matthew et al. 1999). Ya que cada especie tiene una temperatura óptima de crecimiento, siendo para el ballico perenne de 18 ${ }^{\circ} \mathrm{C}$ (Brock y Tilbrook 2000) y al bajar o subir la temperatura cesa su crecimiento (Graming y Stoltenberg 2007). Para el pasto ovillo ocurrió lo contrario al tener el mayor peso por tallo en las estaciones con mayor temperatura y viceversa. La mayor producción de ovillo en las estaciones con mayor temperatura podría deberse a que esta especie tiene una temperatura óptima mayor a ballico perenne con $21^{\circ} \mathrm{C}$ (Brock y Tilbrook 2000), dando como resultado mayor peso por tallo de ovillo en las estaciones donde ballico perenne tuvo el menor peso por tallo, minimizando la estacionalidad de producción de forraje. Esta aseveración se refuerza por investigaciones de evaluación de estas mismas especies (Matthew et al. 1999, Rojas et al. 2017b). Al respecto, Matthew et al. (1996), mencionan que el aumentó en la densidad de tallos por unidad de área ocasiona disminución del peso individual de los tallos, efecto que es explicado por la ley de auto aclareo, confirmada por otros investigadores para gramíneas y leguminosas templadas (Hernández et al. 2000, Rojas et al. 2017a).

\section{Tasa de aparición, muerte y sobrevivencia de tallos}

Las reducciones en el peso por tallo coinciden con el aumento en la tasa de aparición de tallos de ambas especies. Al respecto, Velasco et al. (2007) en praderas de ballico perenne, reportan las mayores tasas de aparición de tallos en verano y menor en otoño. De forma similar, Garduño et al. (2009) al evaluar ballico perenne a diferentes frecuencias e intensidad de pastoreo, registraron la mayor aparición de tallos en verano. Por otra parte, Castro et al. (2013) al evaluar estas mismas especies en asociación reporta para ballico perenne la mayor tasa de mortalidad en la estación de verano, lo que se atribuye al pastoreo selectivo de los ovinos. Mientras que Rojas et al. (2017a) encontraron en pasto ovillo la mayor tasa de sobrevivencia cuando se asocia con $50 \%$ de trébol blanco y menor cuando se suma a la mezcla el ballico perenne. Lo que indica competencia intra e interespecifica por recursos; persistiendo las especies mejor adaptadas al manejo, defoliación y condiciones climáticas (Rojas et al. 2016b). Al 
respecto Rojas et al. (2017b) consideran que las tasas de aparición, muerte y sobrevivencia de tallos de ballico perenne y pasto ovillo depende de la proporción de cada especie en la asociación, estación del año, tiempo de establecimiento de la pradera y factores climáticos. Al respecto Hernández et al. (2000), Graming y Stoltenberg (2007) y Berone et al. (2016) mencionan que la temperatura y la humedad en el suelo, son los principales factores climáticos que influyen en la densidad y tamaño de los tallos, por lo que la tasa de aparición y muerte de estos se relaciona con las condiciones ambientales. Cuando las condiciones son favorables existe constante producción de tallos y mayor peso por tallo, dando como resultado mayor producción de forraje (Rojas et al. 2016a).

\section{CONCLUSIONES}

Los cambios en el peso por tallo de ballico perenne respondieron a cambios en la temperatura, presentando mayor peso en otoño y menor en verano. El peso por tallo en ovillo presentó valores altos en verano y bajos en otoño. La tasa de aparición y muerte de tallos en pasto ballico perenne fue mayor cuando aumenta el porcentaje en la asociación con el siguiente comportamiento: 100:00:00, 70:20:10, 50:00:50, 40:20:40, 20:40:40\% de BP:O:TB. Para el pasto ovillo la tasa de aparición y muerte fue variable, pero depende de la asociación. En ambos pastos la tasa de sobrevivencia disminuyó con el tiempo de establecido.

\section{LITERATURA CITADA}

Arredondo JT, Schnyder H (2003) Components of leaf elongation rate their relationship to specific leaf area in contrasting grasses. New Phytologist 158: 305-314.

Berone GD (2016) Leaf expansion and leaf turnover of perennial C4 grasses growing at moderately low temperatures. Revista de la Facultad de Ciencias Agrarias 48: 69-82.

Brock JL, Tilbrook JC (2000) Effect of cultivar of white clover on plant morphology during the establishment of mixed pastures under sheep grazing. New Zealand Journal of Agricultural Research 43: 335-343.

Castro RR, Hernández GA, Ramírez RO, Aguilar BG, Enríquez QJF, Mendoza PSI (2013) Crecimiento en longitud foliar y dinámica de población de tallos de cinco asociaciones de gramíneas y leguminosa bajo pastoreo. Revista Mexicana de Ciencias Pecuarias 4: 201-215.

Carneiro CS, Do Nascimento JD, Batista EVP (2008) Pastagens: Conceitos básicos, produção e manejo. ViçosaMG. Departamento de Zootecnia. Universidade Federal de Viçosa, San Paulo, Brasil. 115p.

García E (2004) Modificaciones al sistema de clasificación climática de Koppen. 4 ed. Universidad Nacional Autónoma de México. México. 217p.

Garduño VS, Pérez PJ, Hernández GA, Herrera HJG, Martínez HPA, Joaquín TBM (2009) Rendimiento y dinámica de crecimiento estacional de ballico perenne, pastoreado con ovinos a diferentes frecuencias e intensidades. Técnica Pecuaria en México 47: 189-202.

Graming GG, Stoltenberg DE (2007) Leaf appearance base temperature and Phyllichron for common grass and broadleaf weed species. Weed Technology 21: 249-254.

Grünwaldt JM, Guevara JC, Grünwaldt EG, Martínez CE (2015) Cacti (Opuntia sps.) as forage in Argentina dry lands. Revista de la Facultad de Ciencias Agrarias 47: 263-282.

Hernández GFJ, Hernández GA, Ortega JE, Enríquez QJF, Velázquez MM (2015) Comportamiento productivo del pasto ovillo (Dactylis glomerata L.) en respuesta al pastoreo. Agronomía Mesoamericana 26: 33-42. 
Hernández GA, Matthew C, Hodgson J (1999) Tiller size/density compensation in perennial ryegrass miniature swards subject to differing defoliation heights and a proposed productivity index. Grass and Forage Science 54: 347-356.

Hernández GA, Matthew C, Hodgson J (2000) The influence of defoliation height on dry-matter partitioning and $\mathrm{CO} 2$ exchange of perennial ryegrass miniature swards. Grass and Forage Science 55: 372-376.

Lüscher A, Mueller-Harvey I, Soussana JF, Reess RM, Peyraud L (2014) Potential of legume-besed grasslandlivestock systems in Europe: a review. Grass and Forage Science 69: 206-228.

Maldonado PMA, Rojas GAR, Torres SN, Herrera PJ, Joaquín CS, Ventura RJ, et al. (2017) Productivity of orchard grass (Dactylis glomerata L.) alone and associated with perennial ryegrass (Lolium perenne L.) and white clover (Trifolium repens L.). Revista Brasileira de Zootecnia 46: 890-895.

Matthew PNP, Harrington KC, Hampton LG (1999) Management of grazing systems. In: White J, Hodgson J (eds). New Zealand pasture and crop science. Oxford University Press. Melbourne. New Zealand. pp: 153-174.

Matthew C, Hernández GA, Hodgson J (1996) Making sense of the link between tiller density and pasture production. Proceedings of the New Zealand Grassland Association 57: 83-87.

Rojas GAR, Hernández GA, Ayala W, Mendoza PSI, Joaquín CS, Vaquera HH, Santiago OMA (2016a) Comportamiento productivo de praderas con distintas combinaciones de ovillo (Dactylis glomerata L.), ballico perenne (Lolium perenne L.) y trébol blanco (Trifolium repens L.). Revista de la Facultad de Ciencias Agrarias 48: 57-68.

Rojas GAR, Hernández GA, Quero CAR, Guerrero RJD, Ayala W, Zaragoza RJL, Trejo LC (2016b) Persistencia de Dactylis glomerata L. solo y asociado con Lolium perenne L. y Trifolium repens L. Revista Mexicana de Ciencias Agrícolas 7: 885-895.

Rojas GAR, Hernández GA, Rivas JMA, Mendoza PSI, Maldonado PMA, Joaquín CS (2017a) Dinámica poblacional de tallos de pasto ovillo (Dactylis glomerata L.) y ballico perenne (Lolium perenne L.) asociados con trébol blanco (Trifolium repens L.). Revista de la Facultad de Ciencias Agrarias 49: 35-49.

Rojas GAR, Ventura RJ, Hernández GA, Joaquín CS, Maldonado PMA, Reyes VI (2017b) Dinámica poblacional de tallos de ovillo (Dactylis glomerata L.) solo y asociado con ballico perenne (Lolium perenne L.) y trébol blanco (Trifolium repens L.). Revista Mexicana de Ciencias Pecuarias 8: 419-428.

SAS (2009) SAS/STAT ${ }^{\circledR}$ 9.2. User's Guide Release. Cary, NC: SAS Institutelcn. USA. 360p.

Velasco ZME, Hernández GA, González HVA, Pérez PJ, Vaquera HH (2002) Curvas estacionales de crecimiento de ballico perenne. Revista Fitotecnia Mexicana 25: 97-106.

Velasco ZME, Hernández GA, González HVA (2007) Cambios en componentes del rendimiento de una pradera de Ballico perenne, en respuesta a la frecuencia de corte. Revista Fitotecnia Mexicana 30: 79-87. 